The Stata Journal (yyyy)

$v v$, Number $i i$, pp. 1-12

\title{
Nonparametric Instrumental Variable Estimation
}

\author{
Denis Chetverikov \\ Department of Economics \\ UCLA \\ Los Angeles, CA \\ chetverikov@econ.ucla.edu
}

\author{
Dongwoo Kim \\ Department of Economics \\ UCL \\ London, UK \\ dongwoo.kim.13@ucl.ac.uk
}

\author{
Daniel Wilhelm \\ Department of Economics \\ UCL \\ London, UK \\ d.wilhelm@ucl.ac.uk
}

\begin{abstract}
This paper introduces Stata commands [R] npiv and [R] npivcv, which implement nonparametric instrumental variable (NPIV) estimation methods without and with a cross-validated choice of tuning parameters, respectively. Both commands are able to impose the constraint that the resulting estimated function is monotone. The use of such a shape restriction may significantly improve the performance of the NPIV estimator (Chetverikov and Wilhelm 2017). This is because the ill-posedness of the NPIV estimation problem leads to unconstrained estimators that suffer from particularly poor statistical properties such as very high variance. The constrained estimator that imposes the monotonicity, on the other hand, significantly reduces variance by removing nonmonotone oscillations of the estimator. We provide a small Monte Carlo experiment to study the estimators' finite sample properties and an application to the estimation of gasoline demand functions.
\end{abstract}

Keywords: st0001, nonparametric instrumental variable estimation, shape restrictions, monotonicity, endogeneity, regression

\section{Introduction}

Instrumental variable methods are commonly used in economics to achieve identification and consistent estimation of models with endogeneity. Since economic theory does not provide any guidance for how to choose parameterizations of functions of interest, e.g. demand or production functions, it is desirable to avoid imposing such parameterizations when possible. Instead, the nonparametric instrumental variable (NPIV) model does not assume the function of interest is known up to a finite-dimensional parameter:

$$
Y=g(X)+\varepsilon, \quad E[\varepsilon \mid W]=0,
$$

where $Y$ is a scalar dependent variable, $X$ a scalar endogenous explanatory variable, and $W$ an instrumental variable (IV). In practice, however, a researcher often wants to include additional exogeneous covariates $Z$. To avoid the curse of dimensionality in nonparametric estimation, we do not allow the function $g$ to arbitrarily vary with the covariates, but rather assume that they enter the model in an additively separable fashion. We will therefore study the following more general model:

$$
Y=g(X)+\gamma^{\prime} Z+\varepsilon, \quad E[\varepsilon \mid W, Z]=0
$$


We are interested in the estimation of the function $g$ based on a random sample $\left\{\left(Y_{i}, X_{i}, W_{i}, Z_{i}\right)\right\}_{i=1}^{n}$ without imposing any parametric functional form assumptions on $g$. Unfortunately, the nonparametric estimation of $g$ is a very hard statistical problem that requires the solution of a so-called ill-posed inverse problem. These types of estimation problems are well-known to lead to estimators that are very poorly behaved in the sense that they can be extremely variable in finite samples and may converge to the true function $g$ only at a very slow rate. The variance of the NPIV estimator is orders of magnitude larger than that of standard nonparametric regression estimators (based on exogeneous regressors) and, therefore, also much larger than the variance of parametric estimators. More details on the definition and properties of NPIV estimators can be found in Newey and Powell (2003), Hall and Horowitz (2005), Blundell et al. (2007), and Darolles et al. (2011), among others, and in the survey papers Horowitz (2011, 2014).

In many economic applications, the underlying economic theory implies that the function of interest, $g$, should be monotone, e.g. demand, production, or cost functions. Chetverikov and Wilhelm (2017) show that, if this monotonicity condition holds, then constraining the NPIV estimator to satisfy it as well may significantly improve the performance of the estimator in finite samples and, therefore, makes this constraint version of the NPIV estimator more attractive for applied work. One may expect that incorporating the information about the shape in the estimation procedure should at least weakly improve the procedure's performance relative to the one that does not use this information. However, the main point of Chetverikov and Wilhelm (2017) and the current paper is not that there is the possibility of performance improvements, but rather that these may be significant and exploiting them in practice can therefore be very useful. Figure 1 in Chetverikov and Wilhelm (2017) shows the results of a simulation experiment by reporting the square-root of the mean integrated square error ("MISE") of the unconstrained and the constrained NPIV estimators as functions of the sample size. When the series estimator employs four or five series terms and the sample size is around $n=100$, for example, the constrained estimator has a MISE that is only $20 \%$ of that of the unconstrained estimator and, in that sense, is therefore about 5 times more precise. The improvement in MISE from imposing the constraint is visible up to large sample sizes and then eventually disappears.

In this paper, we describe how to use the new Stata command $[\mathrm{R}]$ npiv which implements both the constrained and unconstrained NPIV estimators for a user-chosen number of series terms in the estimator. A second command [R] npivcv, provides the same estimators, but uses a cross-validation criterion to choose the number of series terms in a data-driven fashion.

\subsection{The NPIV Estimator Without Exogenous Covariates}

We first introduce the NPIV estimator in the absence of additional exogeneous covariates $Z$. It is a series estimator that takes the form of the standard two-stage least squares estimator for linear models, except that the nonparametric version considered here does not regress $Y$ on $X$ using $W$ as instrument, but instead regresses $Y$ on a set of transformations of $X$ using a set of transformations of $W$ as instruments. 
Let $\left(Y_{i}, X_{i}, W_{i}\right), i=1, \ldots, n$, be an i.i.d. sample from the distribution of $(Y, X, W)$. To define our estimator, we first introduce some notation. Let $\left\{p_{k}(x), k \geq 1\right\}$ and $\left\{q_{k}(w), k \geq 1\right\}$ be two orthonormal bases in $L^{2}[0,1]$. For $K=K_{n} \geq 1$ and $J=$ $J_{n} \geq K_{n}$, let $p(x):=\left(p_{1}(x), \ldots, p_{K}(x)\right)^{\prime}$ and $q(w):=\left(q_{1}(w), \ldots, q_{J}(w)\right)^{\prime}$ be vectors of basis functions. Define $\mathbf{P}:=\left(p\left(X_{1}\right), \ldots, p\left(X_{n}\right)\right)^{\prime}, \mathbf{Q}:=\left(q\left(W_{1}\right), \ldots, q\left(W_{n}\right)\right)^{\prime}$, and $\mathbf{Y}:=\left(Y_{1}, \ldots, Y_{n}\right)^{\prime}$.

We define two estimators of $g$. The first one is the unconstrained estimator $\widehat{g}^{u}(x):=$ $p(x)^{\prime} \widehat{\beta}^{u}$ which is a linear combination of basis functions with the coefficients estimated by the two-stage least squares optimization problem:

$$
\widehat{\beta}^{u}:=\operatorname{argmin}_{b \in \mathbb{R}^{K}}(\mathbf{Y}-\mathbf{P} b)^{\prime} \mathbf{Q}\left(\mathbf{Q}^{\prime} \mathbf{Q}\right)^{-1} \mathbf{Q}^{\prime}(\mathbf{Y}-\mathbf{P} b) .
$$

This estimator is similar to the one defined in Horowitz (2012) and is a special case of the estimator considered in Blundell et al. (2007). The second one is the constrained estimator $\widehat{g}^{c}(x):=p(x)^{\prime} \widehat{\beta}^{c}$ which is a linear combination of basis functions with the coefficients estimated by the two-stage least squares optimization problem subject to the constraint that the resulting estimator is nondecreasing:

$$
\widehat{\beta}^{c}:=\operatorname{argmin}_{b \in \mathbb{R}^{K}: p(\cdot)^{\prime} b \text { is nondecreasing }}(\mathbf{Y}-\mathbf{P} b)^{\prime} \mathbf{Q}\left(\mathbf{Q}^{\prime} \mathbf{Q}\right)^{-1} \mathbf{Q}^{\prime}(\mathbf{Y}-\mathbf{P} b) .
$$

As indicated in the introduction and further explored in Section 4 the constrained estimator $\widehat{g}^{c}(x)$ may substantially outperform the unconstrained estimator $\widehat{g}^{u}(x)$ if the function $g$ does indeed satisfy the monotonicity constraint.

\subsection{The NPIV Estimator With Exogenous Covariates}

We now introduce two procedures for accommodating the presence of $L$ additional, exogenous covariates $Z$ in the NPIV estimation of $g$. The first procedure gives one-step estimators that are identical to those in (2) and (3) except that the bases $\mathbf{P}$ and $\mathbf{Q}$ are replaced by the enlarged bases

$$
\widetilde{\mathbf{P}}:=[\mathbf{P}, \mathbf{Z}], \quad \widetilde{\mathbf{Q}}:=[\mathbf{Q}, \mathbf{Q} \times \mathbf{Z}]
$$

where $\mathbf{Z}:=\left(Z_{1}, \cdots, Z_{n}\right)^{\prime}$ and $\mathbf{Q} \times \mathbf{Z}$ is the tensor product of the columns of matrices $\mathbf{Q}$ and $\mathbf{Z}$, i.e. the matrix $\mathbf{Q} \times \mathbf{Z}$ is such that its $i$-th row consists of all products of the form $q_{j}\left(W_{i}\right) Z_{i, l}, j=1, \ldots, J$ and $l=1, \ldots, L$. The second procedure gives two-step estimators, which are defined as follows. First, compute the constrained or unconstrained one-step estimator as described above. Let $\widehat{\gamma}$ denote the estimator of $\gamma$ from this first step. Then, remove the covariates from the outcome by defining $\widetilde{Y}:=$ $Y-\widehat{\gamma}^{\prime} Z$ and estimate $g$ by NPIV estimation of $\widetilde{Y}$ on $X$ only, i.e. using (2) or (3). We find that two-step estimators may outperform one-step estimators if the dimension of $Z$ is large.

\section{The npiv and npivcr Commands}

The commands $[\mathrm{R}]$ npiv and $[\mathrm{R}]$ npivcv compute the fitted values of the NPIV estimator of $g$. The former requires the user to specify the number of series terms used in each 
of the two bases $p(x)$ and $q(w)$. The latter constrains the two tuning parameters to be equal and then uses cross-validation to choose them in a data-driven fashion.

The commands require two Stata packages, 'bspline' and 'polyspline'. These can be installed by simply typing 'ssc install bspline' and 'ssc install polyspline.'

When the user does not specify any options, $[\mathrm{R}]$ npiv computes the estimator in (2) or the corresponding one-step estimator if there are additional, exogenous covariates using B-spline bases of degrees 2 and 3, with 2 and 3 knots, for $p(x)$ and $q(w)$, respectively. These default choices and further parameters can be modified through the options described below.

$[\mathrm{R}]$ npivcv is built upon $[\mathrm{R}]$ npiv. Its default choice of bases are also B-splines of degrees 2 and 3 for $p(x)$ and $q(w)$, respectively. It constrains the number of knots to be the same for both bases and then chooses the number of knots by minimizing the cross-validation criterion.

In the absence of monotonicity constraints on $g$, the commands avoid solving the optimization problem in (2) by computing the closed-form two-stage least squares solution of the problem. When the one of the options decreasing or increasing is specified, the commands compute the solution to the constrained optimization problem in (3) to ensure that the resulting estimator is decreasing or increasing, respectively. This optimization problem is implemented using a constrained optimization routine in Mata which typically requires significantly more computation time than the closed-form estimator in the absence of the monotonicity restrictions. As discussed above, however, the gains in precision of the estimator when imposing the constraints may be substantial.

\subsection{Syntax}

The syntax of the commands $[\mathrm{R}]$ npiv and $[\mathrm{R}]$ npivcr is similar:

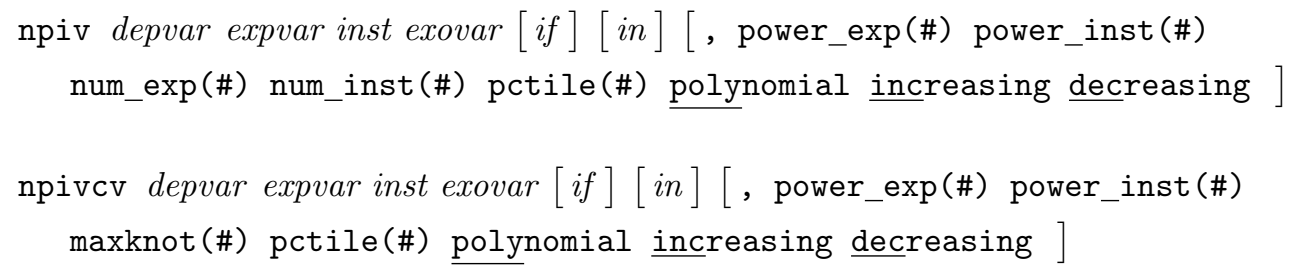

The only difference between the two is that $[\mathrm{R}]$ npiv possesses two additional options for the specification of the number of knots. The four required arguments of the commands are depvar (the outcome variable $Y$ ), expvar (the endogenous regressor $X$ ), inst (the instrumental variable $Z$ ), and exovar (a list of exogenous covariates $W$ ). 


\subsection{Options}

We now describe the options of the two commands. If options are left unspecified, the commands run on the default settings.

power_exp(integer) is a positive integer for the degree of the spline basis for the endogenous regressor. The default is 2 .

power_inst (integer) is a positive integer for the degree of the spline basis for the instrument. This number needs to be equal to or larger than power_exp. The default is 3 .

num_exp(integer) is a positive integer greater than 1 for the number of knots of the spline basis for the endogenous regressor. The default is 2 . The user need not specify this if polynomial is used.

num_inst (integer) is a positive integer greater than 1 for the number of knots of the spline basis for the instrument. The default is 3 . The user need not specify this if polynomial is used.

maxknot (integer) is a positive integer for maximum number of knots to be considered in the cross-validation procedure. With a sample size $N$ and the option maxknot(k) the cross-validation procedure evaluates the performance of the NPIV estimator with numbers of knots from 3 to $\max \left(N^{1 / 5}, k\right)$ and executes the NPIV regression with the optimal number of knots from that range. If the option polynomial is used, then maxknot specifies the maximum power of the polynomial to be considered. 5 is the default.

pctile(integer) specifies the domain of the endogenous regressor over which the NPIV estimator of $g$ is to be computed. This needs to be a positive integer smaller than 50. For a given value $k$, the NPIV estimator is computed at fine grid points within the $k$-th and the $(100-k)$-th percentiles of the empirical distribution of $X$. The default is 5 .

polynomial specifies the type of both bases $p(x)$ and $q(w)$ to be power polynomials. Choices of numbers of knots are ignored under this option. Shape restrictions cannot be imposed for this basis and an error message is generated if this option is used together with decreasing or increasing.

increasing imposes that the NPIV estimator is an increasing function of the endogenous regressor. If this option is specified, the basis $p(x)$ is forced to be quadratic B-spline and the option power_exp(integer) is not used. An error occurs when this option is used together with one of the options decreasing or polynomial. The basis $q(w)$ for the instrument is also restricted to be a B-spline, but the power and number of knots can be freely chosen through power_inst(integer) and num_inst(integer).

decreasing imposes that the NPIV estimator is a decreasing function of the endogenous regressor. The same restrictions as for increasing apply to this option. 


\subsection{Saved results}

The commands npiv and npivcv each generate several output variable in the Stata data space. There are two variables called npest and grid. npest contains the fitted values of the NPIV estimator of $g$ over the range indicated by pctile(integer). grid contains a grid of values of expvar over which the estimator is evaluated. In addition, the commands generate variables of the form $g_{-} r_{-} i_{-} d *, e_{-} x_{-} p_{-} v *$, and $i_{-} n_{-} s_{-} t *$ where "**" is replaced by the index of a series term. These three variables contain the values of the series functions evaluated at grid, at expvar, and at inst, respectively. When npiv is run a second time, the results in $g_{-} r_{-} i_{-} d *, e_{-} x_{-} p_{-} v *$, and $i_{-} n_{-} s_{-} t *$ are copied to new variables $g_{-} r_{-} i_{-} d_{-} o l d *, e_{-} x_{-} p_{-} v_{-} o l d *$, and $i_{-} n_{-} s_{-} t_{-} o l d *$, and the new results are stored in $g_{-} r_{-} i_{-} d *$, $e_{-} x_{-} p_{-} v *$, and $i_{-} n_{-} s_{-} t *$. The behavior of npivcv is similar except that old results are stored in variables called $g_{-} r_{-} i_{-} d_{-} c v_{-} o l d *, e_{-} x_{-} p_{-} v_{-} c v_{-} o l d *$, and $i_{-} n_{-} s_{-} t_{-} c v_{-} o l d *$.

Both commands are of the e-class. The following results are stored in e():

\begin{tabular}{|c|c|c|c|}
\hline Scalars & & & \\
\hline$e(N)$ & number of observations & e (powerexp) & power of basis for $X$ \\
\hline e (powerinst) & power of basis for $W$ & e(numexp) & \# of knots of basis for $X$ \\
\hline e(numinst) & $\#$ of knots of basis for $W$ & $e(p c t)$ & percentile \\
\hline$e(x \min )$ & min of $X$ in domain & $e(x \max )$ & $\max$ of $X$ in domain \\
\hline e(zmin) & min of $Z$ in domain & $e(z \max )$ & $\max$ of $Z$ in domain \\
\hline e (gmin) & min of grid & e (gmax) & max of grid \\
\hline e (optknot) & $\begin{array}{l}\text { on which g evaluated } \\
\text { optimal number of knots }\end{array}$ & e(maxknot) & $\begin{array}{l}\text { on which } g \text { evaluated } \\
\text { maximum knots to be evaluated }\end{array}$ \\
\hline Macros & & & \\
\hline $\mathrm{e}(\mathrm{cmd})$ & name of command & & \\
\hline e(depvar) & name of $Y$ & e(expvar) & name of $X$ \\
\hline$e$ (inst) & name of $Z$ & e(exovar) & list of exogenous regressors \\
\hline e(basis) & type of spline basis & $e(t i t l e)$ & nonparametric IV regression \\
\hline e(shape) & type of shape restriction & & \\
\hline $\begin{array}{c}\text { Matrices } \\
\text { e(b) }\end{array}$ & oefficient vector of basis & & \\
\hline
\end{tabular}

In the presence of additional, exogenous covariates $Z$ of dimension $k$, the estimates of $\gamma$ correspond to the last $k$ elements of the coefficient vector in e(b).

\section{Example: Estimating Gasoline Demand Functions}

In this section, we illustrate the use of the NPIV estimation commands by applying them to the estimation of gasoline demand functions on the household level as in Blundell et al. (2012, 2016); Chetverikov and Wilhelm (2017). We consider the partially linear specification in (1) where $Y$ is annual log-gasoline consumption (log_q), $X$ denotes log-price of gasoline ( $\log \mathbf{p}$ ), and the instrument $W$ is distance to major oil platform (distance_oil1000). We include additional controls $Z$ such as household characteristics, urbanity, population density, and availability of public transit, among others. The controls are listed in the local macro 'exolist'. The data set is from the 2001 National Household Travel Survey and the sample size is 4,812. See Blundell et al. (2012) for more details on the data set, sample selection and definition of the variables. Price and 
income elasticities are constant in a fully linear model, but it is more likely that those elasticities vary with price and income levels. By using the nonparametric function $g$, we allow for non-constant price elasticity. We focus on three subsamples in which household income levels are within \pm 0.5 (in log) of $\$ 72,500$ (high), $\$ 57,500$ (middle) and $\$ 42,500$ (low).

Figures 1 and 2 show the unconstrained and constrained (with a decreasing shape) NPIV estimates of $g$. In both estimation procedures, a quadratic B-spline basis with 3 knots is used for $p(x)$ and a cubic B-spline with 10 knots for $q(w)$. The NPIV demand function is estimated on price levels from 5th to 95th percentiles of the given data.

We now describe how to produce these results using the command npiv for the middle income group. First, consider estimation of the one-step unconstrained NPIV estimator. The following code defines the parameters of the B-spline basis, drops observations outside the middle income group, defines the list of additional exogenous covariates, then executes the npiv command, and finally stores the estimated demand function in the variable one_step:

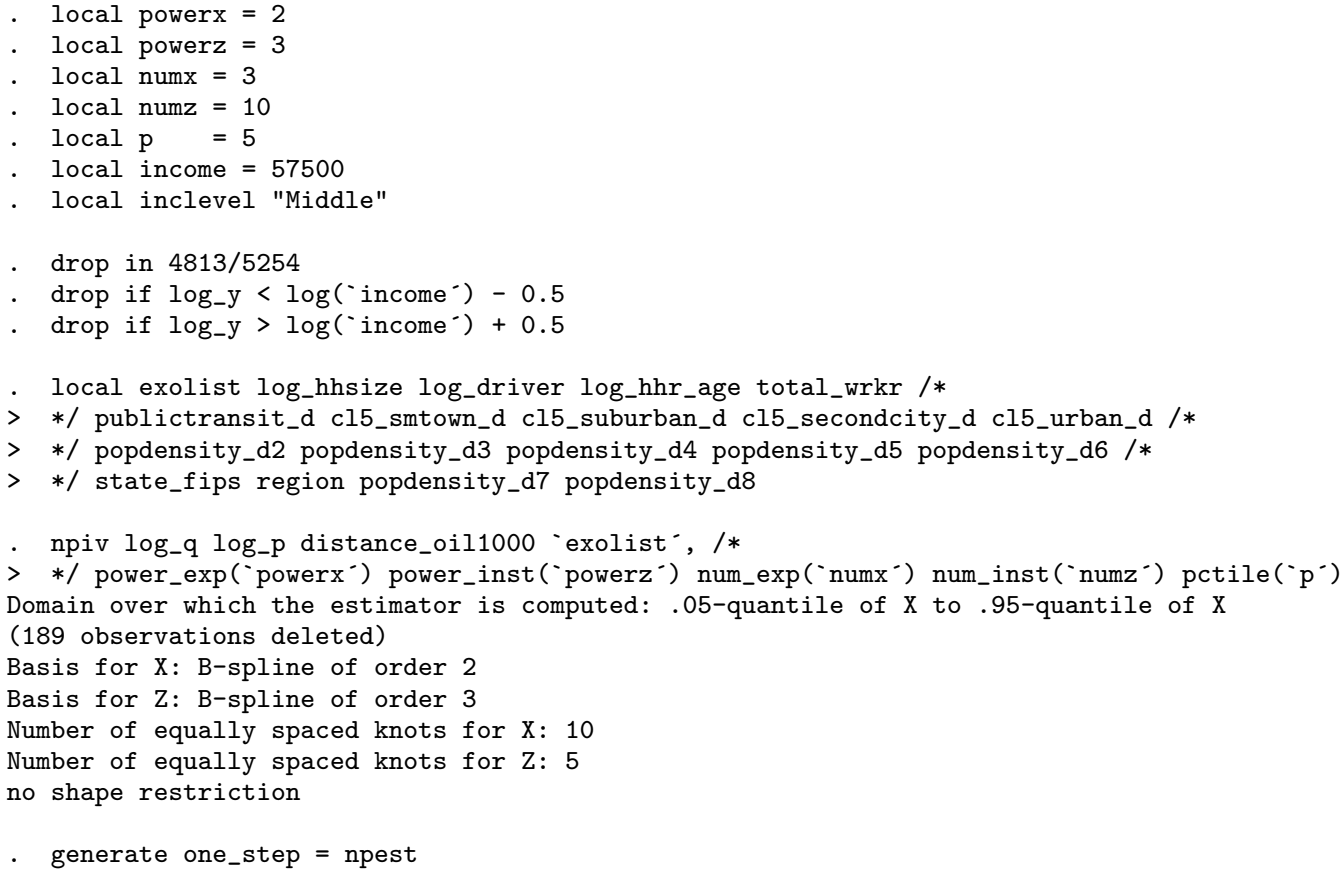

For the estimation of the two-step unconstrained NPIV estimator, we need to subtract the index of covariates, $\widehat{\gamma}^{\prime} W$, from the outcome. The following code estimates the index coefficients $\widehat{\gamma}$ and stores the difference between the outcome and the index of covariates in Y tilde.

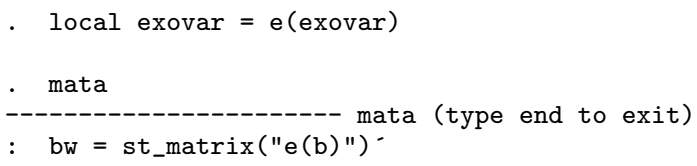




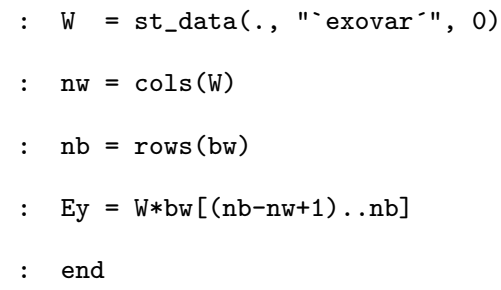

. getmata Ey, force

- quietly generate Y_tilde = log_q - Ey

The two-step unconstrained NPIV estimator is then computed as the unconstrained NPIV estimator without additional covariates, using Y_tilde as the new outcome:

- npiv Y_tilde log_p distance_oil1000, /*

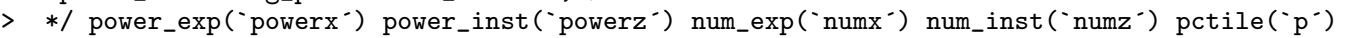

- mata

------------------- mata (type end to exit)

: npest $=$ st_data(., "npest", 0)

: one_step $=$ st_data $(.$, "one_step", 0)

: onestep $=$ one_step $:+$ bw $[n b-n w+1] * \log \left(\right.$ 'income $\left.^{-}\right)$

: twostep $=$ npest $:+$ bw $[n b-n w+1] * \log \left({ }^{\text {'income }}{ }^{\prime}\right)$

: end

Finally, we recover the one-step and two-step estimates from mata and plot them together in the same graph:

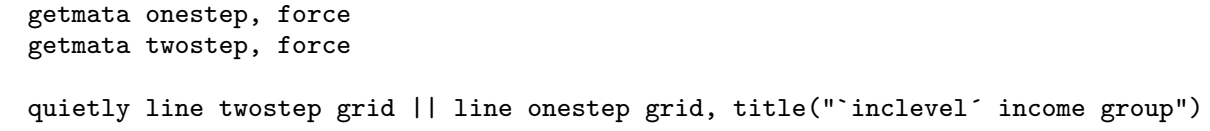

The resulting graph is the second one in Figure 1. The other two graphs for the lower and upper income group are generated in a similar fashion. Similarly, the constrained estimators are constructed similarly, making use of the option decreasing of the npiv command.

\section{Monte Carlo Simulation}

In this section, we conduct a small simulation experiment to study the finite sample properties of the NPIV estimators discussed in this paper and to show that imposing monotonicity constraints can substantially improve the performance of the estimators (provided the population function $g$ also satisfies the monotonicity constraint). 


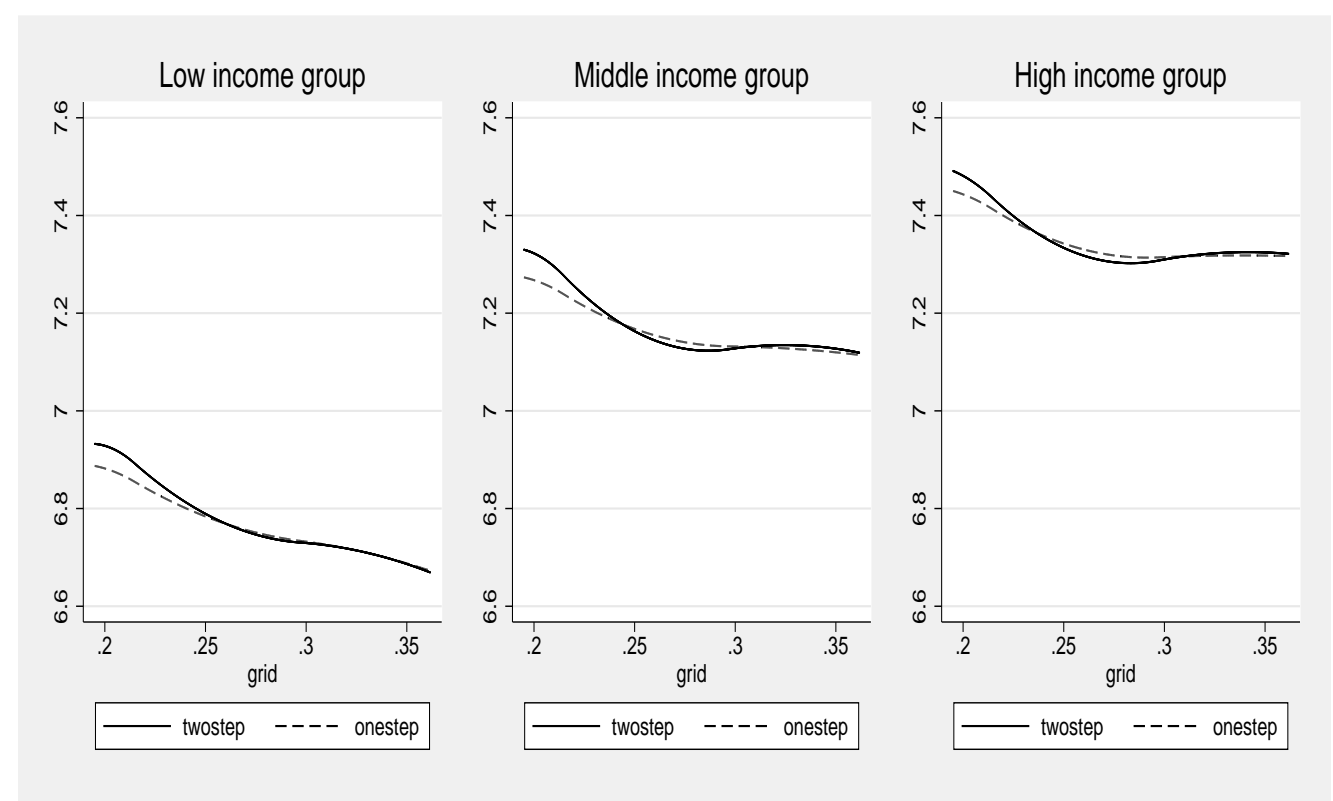

Figure 1: Unconstrained NPIV estimates of $g$ in the three income groups using the one-step and two-step estimators to accommodate additional covariates.

Consider the following data generating process:

$$
\begin{aligned}
& {\left[\begin{array}{l}
\varepsilon \\
\eta
\end{array}\right] } \sim N\left(\left[\begin{array}{l}
0 \\
0
\end{array}\right],\left[\begin{array}{cc}
1 & 0.5 \\
0.5 & 1
\end{array}\right]\right), \\
& W \sim N(0,1), \quad X=2 W+\eta \\
& Y=g(X)+\varepsilon \quad \text { where } \quad g(X)=\frac{\exp (X / 2)}{1+\exp (X / 2)}
\end{aligned}
$$

$Y, X$ and $W$ denote the outcome, the endogenous regressor, and the instrument respectively. $\eta$ is the endogenous component of $X . W$ is independent of $\varepsilon$ and $\eta$ by construction. We generate 1,000 Monte Carlo samples of size 100 and of size 800 .

First, we compute the unconstrained NPIV estimator:

. npiv Y X W, power_exp(2) power_inst(3) num_exp(3) num_inst(3) pctile(1)

Second, we compute the constrained NPIV estimate that imposes that the estimator is increasing:

. npiv Y X W, power_exp(2) power_inst(3) num_exp(3) num_inst(3) pctile(1) increasing

Figures 3 and 4 show the true $g$ function and the NPIV estimate averaged across the 1,000 samples. Red lines show bands that indicate the variability of the estimator. The lower and upper band were generated by subtracting and adding 2 -times the empirical standard deviations from the average estimate at each grid point. 

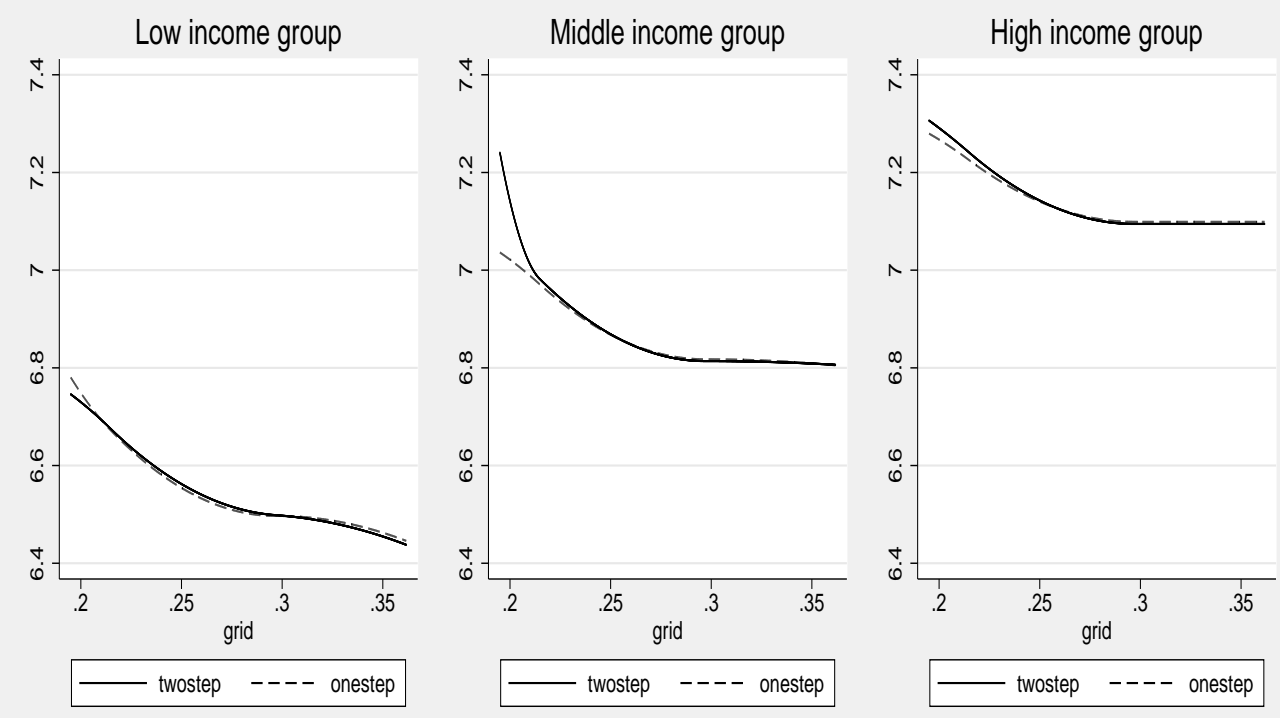

Figure 2: Constrained NPIV estimates of $g$ in the three income groups using the one-step and two-step estimators to accommodate additional covariates.

For both sample sizes, the two estimators incur only a small bias, but the constrained NPIV estimator is much less variable than the unconstrained one. The difference in variability decreases as the sample size increases. Eventually, as the sample size becomes large enough the constrained and unconstrained estimators will be equal to each other, although this may happen only for extremely large samples. These findings are consistent with the Monte Carlo simulation results in Chetverikov et al. (2017). 

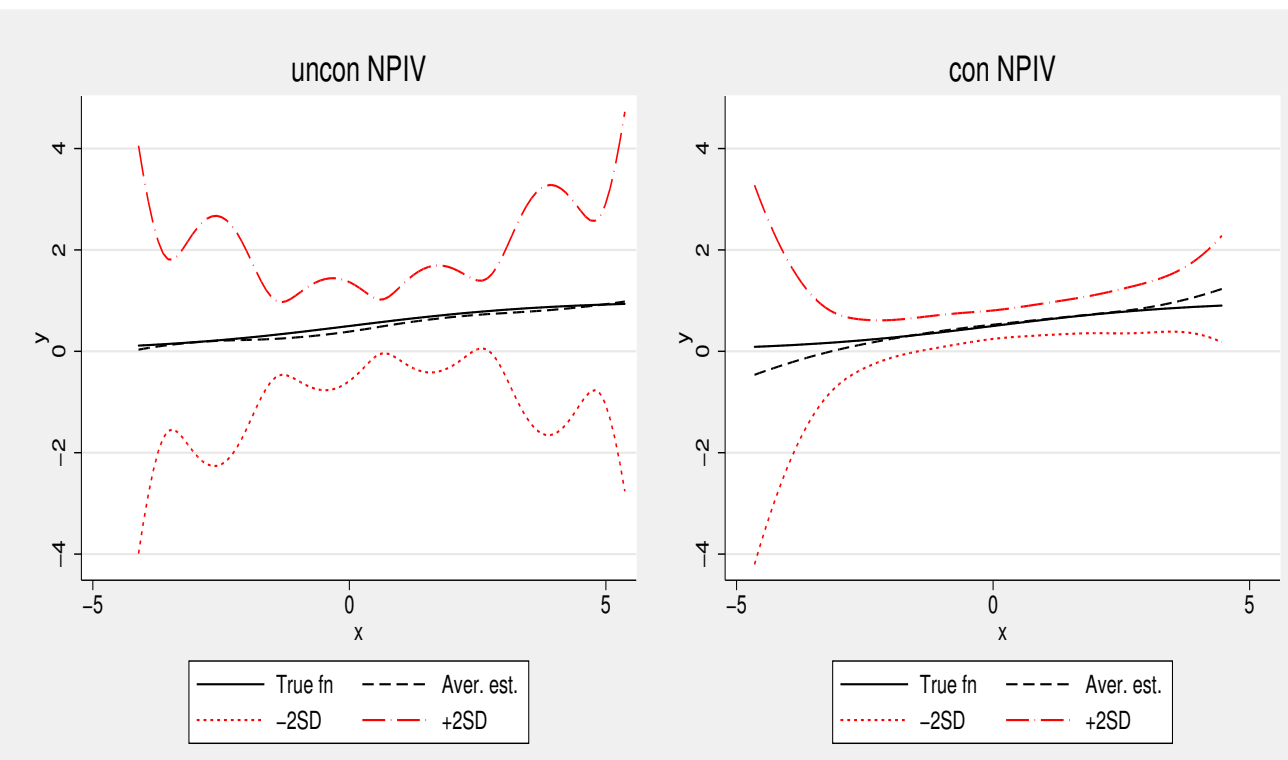

Figure 3: Monte Carlo simulation results on small samples
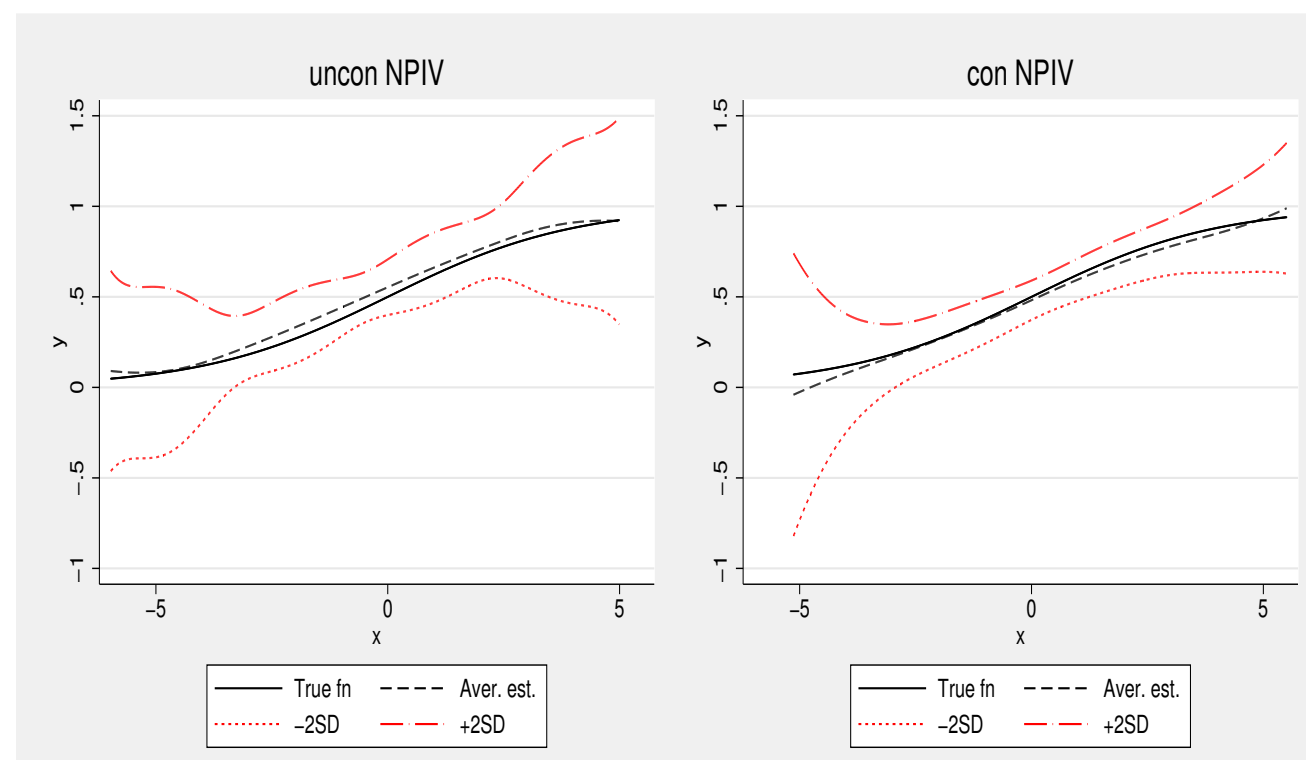

Figure 4: Monte Carlo simulation results on large samples 


\section{References}

Blundell, R., X. Chen, and D. Kristensen. 2007. Semi-Nonparametric IV Estimation of Shape-Invariant Engel Curves. Econometrica 75(6): 1613-1669.

Blundell, R., J. Horowitz, and M. Parey. 2016. Nonparametric Estimation of a Nonseparable Demand Function under the Slutsky Inequality Restriction. Review of Economics and Statistics forthcoming.

Blundell, R., J. L. Horowitz, and M. Parey. 2012. Measuring the price responsiveness of gasoline demand: Economic shape restrictions and nonparametric demand estimation. Quantitative Economics 3(1): 29-51.

Chetverikov, D., D. Kim, and D. Wilhelm. 2017. An Adaptive Test of Stochastic Monotonicity. Technical report.

Chetverikov, D., and D. Wilhelm. 2017. Nonparametric Instrumental Variable Estimation Under Monotonicity. Econometrica 85(4): 1303-1320.

Darolles, S., Y. Fan, J. P. Florens, and E. Renault. 2011. Nonparametric Instrumental Regression. Econometrica 79(5): 1541-1565.

Hall, P., and J. L. Horowitz. 2005. Nonparametric Methods for Inference in the Presence of Instrumental Variables. The Annals of Statistics 33(6): 2904-2929.

Horowitz, J. L. 2011. Applied Nonparametric Instrumental Variables Estimation. Econometrica 79(2): 347-394.

- 2012. Specification Testing in Nonparametric Instrumental Variable Estimation. Journal of Econometrics 167(2): 383-396.

. 2014. Ill-Posed Inverse Problems in Economics. Annual Review of Economics 6: $21-51$.

Newey, W. K., and J. L. Powell. 2003. Instrumental Variable Estimation of Nonparametric Models. Econometrica 71(5): 1565-1578.

\section{About the authors}

Denis Chetverikov is an Assistant Professor in the Department of Economics at University of California at Los Angeles.

Dongwoo Kim is a $\mathrm{PhD}$ candidate in the Department of Economics at University College London.

Daniel Wihelm is a Lecturer in Economics at University College London, a staff member of the ESRC Centre for Microdata Methods and Practice (CeMMAP), a Research Fellow at the Institute for Fiscal Studies, and a Research Fellow at the Centre for Research and Analysis of Migration (CReAM). 